\title{
Transforming the European Union into a United Europe: The Role of Communication between Formation and Information
}

Rudi Papa

“Sapienza” University of Rome, Department of Political Science; rudi.papa@uniroma1.it

Diva Ricevuto

Associazione Sulle Regole; divaricevuto@gmail.com

Dario Quattromani

“Roma Tre University”, Department of Political Science; dario.quatromani@uniroma3.it

Maria Pia Di Nonno

mariapiadinonno@gmail.com

Doi:10.5901/mjss.2015.v6n5s1p376

\begin{abstract}
The current European Union is a global actor in search of an effective communication strategy. This institution represents the momentary result of a long process, which started even before the end of the Second World War, and it is now facing a critical moment: whilst aware of the great achievements and the need to obtain increasingly more important ones, it does not seem like to be able at informing its citizens. Guided by article 11 of the Charter of Fundamental Rights and by Prot. 29, the European Union focuses its attention not only on in-formation but also on the formative role that this can have for citizens. The subject of this article is a brief analysis of the main instruments adept for European information, mostly on the web (mainly consulted by insiders and less by others), along with some reflections on the chance of proposing the making of informed participation available to the European citizens: how information (which creates knowledge) includes training (which creates consciousness) in a virtuous dynamic game. An excursus on the vision of the society of communication, as forecasted by trend analysts of the eighties will help comprehending the relevance of a Europe on the run, where increasing technological projects are re-designing its social structure. The society of knowledge will be able to change the European citizen and build up an even more solid community only if focusing its energies on memory, an indispensable baggage, invaluable experience common to all European citizens. The adult generations are risking exclusion, despite the cultural richness they own: the reduction of the digital divide should be addressed also to them, as well as an engine for the new generations, seeking an harmony that may only be fostered with knowledge creating greater inclusion. It is still free the way to use the new means of communication, that everyone can see as an obstacle or an opportunity.
\end{abstract}

Keywords: Europe, Formation, Communication, European Citizen, Founding Mothers.

\section{Introduzione}

L'Unione Europea è ormai attore globale in questioni di politica estera ed è attivo nella promozione dei propri valori e obiettivi, sia all'interno che all'esterno dell'Unione stessa con la PESC (Politica Estera e di Sicurezza Comune).

Poiché la comunicazione è uno degli strumenti per potenziare la democrazia è necessario che si elabori una strategia coerente in materia di informazione.

I canali media europei, determinanti per comprendere e dibattere le ragioni che uniscono e quelle che dividono, possono rendere l'Europa più omogenea nella comprensione della politica, delegata con il voto, mostrando le attività istituzionali.

L'informazione di e sull'Europa non ha la diffusione capillare che riscontriamo sui temi di interesse nazionale e internazionale, per i quali possiamo attingere a molte fonti.

Come ovviare a questo problema? Come far uscire l'informazione da una nicchia per addetti ai lavori e offrirla a tutti i cittadini europei? 
L'informazione, quando è completa, diventa un metodo di formazione continua, uno stimolo al dibattito e uno strumento di evoluzione del pensiero. Accresce la conoscenza (in-formazione/formazione) e si crea la coscienza (consapevolezza).

L'art. 11 della Carta dei diritti fondamentali dell'UE del 2000 sulla "libertà di espressione e d'informazione" sottolinea il diritto di tali libertà agli Stati membri. In particolare il Protocollo n. 29, recita "il sistema di radiodiffusione pubblica negli Stati membri è direttamente collegato alle esigenze democratiche, sociali e culturali di ogni società, nonché all'esigenza di preservare il pluralismo dei mezzi di comunicazione".

\section{Strumenti della Comunicazione Per L'in-Formazione}

Uno degli obiettivi principali dell'UE è quello di creare un'identità europea, l'identità del cittadino europeo. Questo tema dalle molte sfaccettature lo affrontiamo da due punti di vista: quello della formazione (coscienza) e quello dell'informazione (conoscenza).

Al fine di accrescere conoscenza e coscienza europea dei cittadini nel vecchio continente, vediamo gli strumenti a loro disposizione, sia quando ricerchino informazioni, sia quando le ricevano. Lo stato dell'arte dell'informazione europea è per pochi sebbene esistano siti internet ufficiali delle istituzioni europee sui quali è possibile informarsi in merito alle attività dell'Unione. I canali multilingue (vedi Euronews, EurActiv e VoxEurop, che ha sostituito Presseurop), offrono una informazione di livello accettabile.

II servizio pubblico nazionale italiano ha creato "Alfabeto Europa" -per portare l'utente 'alla scoperta dell'Europa'1, della sua storia, delle sue istituzioni, della sua economia e delle opportunità per i cittadini europei- e "Cantiere Europa", anch'esso RAl, -per seguire il semestre di presidenza italiano e per coinvolgere i cittadini "da ogni angolo dell'Europa e del mondo"2. Questi alcuni esempi di iniziative spesso ad opera di giornalisti privati che pubblicano news di respiro europeo.

Le informazioni sull'Europa si concentrano principalmente sul web, accessibile a pochi a causa del digital divide, e sono ricercate solo da chi ha interesse alla materia, mentre sono trascurate dagli altri creando un gap crescente tra chi è informato e chi lo è meno, o lo è per nulla.

Citiamo ad esempio il dibattito tra candidati alla Presidenza della Commissione, aprile e maggio 2014, passato praticamente sotto silenzio sulla maggior parte delle reti televisive nazionali eccezion fatta per quello del 15 maggio, condotto da Monica Maggioni per Rainews24.Nell'agenda digitale "Horizon 2020" - una delle sette iniziative faro della strategia "Europa 2020" -che fissa gli obiettivi per la crescita nell'Unione Europea (UE) da raggiungere entro il 2020- posa l'attenzione sul potenziale delle tecnologie dell'informazione e della comunicazione (TIC) per favorire innovazione, crescita economica e progresso comuni. Al fine di migliorare la strategia comunicativa dell'Europa il Parlamento Europeo, già nell'aprile 2014, un gruppo di parlamentari della Commissione Affari Esteri si occupò di redigere la "Raccomandazione al Consiglio, alla Commissione e al Servizio Europeo per l'azione esterna" in merito al ruolo della comunicazione audiovisiva visto come strumento di rappresentanza dell'UE e dei suoi valori.

II documento ritiene le trasmissioni radiotelevisive e i servizi audiovisivi online "modalità efficienti ed economicamente vantaggiose per raggiungere il pubblico in Europa e nei paesi terzi e costituiscono la fonte principale di informazione per la maggior parte dei cittadini all'interno e all'esterno dell'Unione". Ricordiamo il canale Euronews, finanziato interamente dalla Commissione europea, dal 2005, che trasmette in 13 lingue, raggiunge circa 155 paesi ed viene compreso dal $53 \%$ della popolazione mondiale.

La raccomandazione, in particolare, riguardava l'implementazione e l'utilizzo di piattaforme di comunicazione per raggiungere i paesi terzi e "promuovere i valori base dell'Unione" -quali diritti umani, valori democratici, pluralità di pensiero e di informazione- tramite una "strategia di comunicazione audiovisiva dell'UE coerente e solida nel quadro delle relazioni esterne dell'Unione, promuovendo così la libertà di espressione e il pluralismo dei mezzi di comunicazione nonché sostenendo e rafforzando la democrazia e i diritti umani in Europa e nei paesi terzi; fare di tale strategia uno strumento integrato nella PESC".

Far conoscere l'Unione Europea ai suoi cittadini, ai vicini orientali prossimi all'Unione, ma anche ai paesi di lingua araba e quelli africani, e diffondere le idee democratiche, fondamento dell'Europa, con i mezzi di comunicazione europei, verso società in cui, a volte, la libertà di informazione viene limitata. Raggiungere anche risultati concreti e duraturi informando soprattutto chi non sa di dover essere informato. Citiamo a tal proposito l'esperienza dei sondaggi deliberativi, che nel 2009 ha raggiunto gli allora 27 paesi membri dell'Unione Europea con EuroPolis. Si trattò d'un

\footnotetext{
${ }^{1}$ http://www.raistoria.rai.it/europa/.

${ }^{2}$ http://www.europa.rai.it/.
} 
progetto che ebbe inizio nel settembre del 2008, durò 24 mesi, e che esplorò l'eventuale deficit democratico dei cittadini dell'Unione.

La valutazione degli esiti dei cosiddetti "sondaggi deliberativi" a differenza di quelli tradizionali, cerca di "delineare un quadro di ciò che l'opinione pubblica penserebbe se si concentrasse sugli stessi temi in condizioni ideali e, in particolare, se fosse, riguardo a tali questioni, più adeguatamente informata"3.

EuroPolis cercò di capire se una maggiore informazione sull'organizzazione, il processo decisionale, le questioni di policy, nonché la conoscenza dei punti di vista dei cittadini degli altri Stati membri avrebbe potuto cambiare il comportamento politico e sociale dei cittadini europei ed avere effetti sulla partecipazione politica e l'affluenza alle urne.

Uno dei problemi principali per la realizzazione di tali eventi riguarda i costi, ma "if you think research is expensive, just try disease". La peculiarità dell'esperimento è stata la possibilità di sperimentare, da parte dei partecipanti, dei comportamenti da cittadini ideali dell'Europa, un'esperienza senza precedenti nella storia dell'opinione pubblica del vecchio continente. Tale progetto voleva creare una sfera pubblica paneuropea in cui potesse essere facilitata l'espressione della "Voice of the People"4.

\section{Dall'informatica alla Societa' della Conoscenza}

Il grande compito e la grande ambizione della frontiera dell'in-formazione su cui l'Europa ha, da subito, puntato è il percorso indicato, già negli anni ottanta, da analisti globali quali Servan-Schreiber ed Alvin Toffler, che condivisero l'idea che l'impiego a tutti i livelli dell'informatica avrebbe portato verso un appianamento dei contrasti nord-sud. Questo si è avverato in Europa sotto molti aspetti. Toffler vide anche che si sarebbe realizzata una forte decentralizzazione del potere ed una veloce ristrutturazione dei rapporti familiari.

John Naisbit dal canto suo, in Megatrend e in Megatrend 2000, lesse un miglioramento della qualità di vita di tutti $\mathrm{i}$ cittadini in possesso degli strumenti informatici e disse che la società evolvendosi con tempi così accelerati non potesse essere analizzata, da quel momento in poi, che sulla base di dati previsionali. Alain Minc, negli stessi anni, scrisse che sarebbe stata necessaria una riprogettazione della struttura sociale su criteri nuovi al passo con i mutamenti tecnologici. La società della Conoscenza, come si cominciò efficacemente a chiamarla dal ' 90 in poi, per la grande movimentazione di informazioni che portò con sé, ebbe uno sviluppo così rapido da creare uno stato di incertezza ma fu destinata ad entrare nel tessuto sociale come l'elettricità 0 il telefono, e a creare nuovi habitus per vivere 0 lavorare. Furono allora progettati i primi terminali interattivi ad uso della massa, per luoghi pubblici, e poi ci si volse ad un incremento dell'informatica individuale e dell'utenza domestica, coinvolgendo i cittadini ancora inconsapevoli e rendendoli da subito attivi formulatori di nuove proposte di utilizzo.

\section{La Societa' della Conoscenza e la Memoria Stimolano un Nuovo Cittadino Europeo}

"Peculiare dell'Europa è la diversità dei popoli che la compongono", scrive Julia Kristeva, "che molti ambiscano abitarla e che, per questo, donne e uomini in carne e ossa cerchino di raggiungerla per accedere ad una vita migliore cui hanno pieno diritto".

La memoria culturale è comune in questo continente, innegabilmente legato nel profondo.

Per millenni, quella che chiamiamo Europa "percepì se stessa, non come una parte del mondo, ma come il mondo stesso" aggiunge la Kristeva. Una cultura comune europea esiste e ad essa dobbiamo riferirci senza esitazione. L'Europa è sempre stata unita, come il mondo del resto. Gli antichi romani la percorsero a piedi. Prima dell'avvento delle macchine a vapore, veniva solcata in lungo e in largo in carrozza e a cavallo. Con gli uomini si sono mosse anche le idee e il sapere che si è moltiplicato e raffinato fino ad oggi. L'Europa è ed ha un patrimonio inestimabile. È stata teatro dell'emancipazione di popoli, di gruppi, di genere. Ha vinto su inquisizione, colonialismo, Shoah. Con il suo patrimonio culturale e artistico è un riferimento federatore ineludibile. II pregiudizio e le idee dividono e si alimentano con le crisi che tormentano i cittadini europei, tuttavia, essi non mettono in discussione la propria appartenenza all'Europa. II voto, unito alla forte astensione, che si è diretto verso le formazioni antieuropee nel 2014, così come il successivo referendum inglese, può addirittura essere letto come una conferma di ciò: il grido di dolore che fa eco e che smaschera le posizioni opportunistiche di chi approfitta, per proprio tornaconto e privilegio, d'entrare a far parte del sistema comunitario utilizzandolo senza pudore. Saremmo superficiali, però, a non considerare il disagio europeo che è concreto e che confonde. È tempo di aprire i sensi alla comprensione della complessità della condizione umana in Europa. È tempo di

\footnotetext{
${ }^{3}$ http://www.caffeeuropa.it/demdelib/284fishkin.html.

${ }^{4}$ http://www.caffeeuropa.it/demdelib/284fishkin.html
} 
lavorare alla ri-costruzione del sentire europeo alla luce della mutata società del terzo millennio.

È tempo di mirare a comporre gli intessi profondi dei cittadini europei così diversi e contaminati. Settant'anni di pace in Europa hanno donato una dimensione nuova a questo continente che desidera allargarsi anche a paesi fratelli, pur se meno stabili. di qualità.

La consapevolezza spontanea delle Madri e dei Padri Fondatori è giunta a maturazione e siamo pronti per il salto

Va superata la frontiera della consapevolezza nel sentirci, oltre che chiamarci, Europei, per non essere in balia di mosse dettate dalle migliori intenzioni in un quadro fuori regia. Talvolta si raggiungono buoni obiettivi anche in condizioni faticose di necessità ma la differenza la fa il prezzo che si paga. Gli errori commessi durante un percorso progettato, invece, si possono riparare prima che siano enormi. II cittadino europeo è portatore di valori individuali, catalizzatore di interessi collettivi. II patrimonio di memoria gli fa da impulso attraverso la filosofia, le scienze umane, la secolarizzazione, le costituzioni, le istituzioni, e lo aiuta a capire. L'Europa è in cammino, l'identità europea in divenire, viene da lontano. Un drappello di pochi è partito all'inizio del secolo breve ed è ancora in cammino. Durante il percorso si sono aggiunti altri e poi sempre di più. La memoria è un bagaglio leggero ma anche una solida base su cui costruire un futuro sempre migliore. Poggiando sulla memoria questa magnifica e progressiva sorte non si arresterà mai. II metodo a questo punto farà la differenza, porterà ispirazione alle nuove generazioni. Come lungimiranti furono i nostri predecessori che non caddero preda dello scoramento o del disimpegno e vissero un presente difficile come sono difficili tutti i presenti dell'umanità. Attrezziamoci a scongiurare i dolori che si pongono nuovamente a poche miglia da noi perché il mare non ci separa più, può invece unirci, come ci unisce di fatto. Va pensato un senso nuovo dell'educazione: 'accademica', 'informale' e 'continua'. Vanno sostenute intere generazioni che si trovano, quasi improvvisamente, a rischio esclusione non per obsolescenza delle proprie competenze o cultura ma per via dei modi di utilizzarle. Bisogna aiutare le giovani generazioni che non possono spiccare il volo se le precedenti non volano esse stesse. In passato la storia ha mostrato numerosi casi di scollamento tra evoluzione tecnica e società, e se il fattore tempo sospinge sempre in avanti, è il progresso intellettuale che deve sostenere la prova col costruire una società de-verticalizzata, reticolare, orizzontale. E' il metodo l'unico elemento in grado informare le nostre scelte e dare risposte di innovazione, di inclusività, di sostenibilità.

\section{Comunicazione, Dis-Informazione e Potere}

Viviamo davvero nell'era della comunicazione? Gli apocalittici ritengono di vivere in quella della dis-informazione. Siamo immersi in una tempesta di informazioni, notizie e dati. Alcuni pensano che il nostro livello di conoscenza sia inferiore a quello dei nostri avi poiché abbiamo una minore abitudine a memorizzare. È una contraddizione? Niente affatto. Avere molte informazioni confonde, perché si agisce come ci si trovasse costantemente a un bivio. In questa immensa offerta le scelte sono complesse e devono tenere conto di molti fattori contemporaneamente. Similmente accade per i mass media: televisione, giornali, radio e social media (ad esempio Facebook e Twitter) potrebbero essere strumenti per acquisire cultura mentre a volte abbassano la qualità del dibattito.

Ciò accade perché abbiamo mutato modo di pensare. Si legge di più ma meno a livello qualitativo. Centoquaranta caratteri su Twitter bastano a spiegare la nostra vita, le nostre decisioni o importanti eventi politici e culturali. Crediamo che seguire un quotidiano su Twitter sia equivalente a leggere un buon articolo. Un tweet non sostituisce ma stimola il desiderio di curiosità. I nuovi mezzi di comunicazione sono innovativi e devono essere usati in modo nuovo.

Sono un ponte verso la cultura e non un percorso a senso unico. Viviamo in una società liquida, come evidenzia il sociologo Zygmunt Bauman, dove tutto scorre veloce -secondo il pensiero di un classico come Eraclito- senza il tempo per riflettere. Sempre connessi, produciamo una grande quantità di messaggi e condividiamo meno i valori nella vita reale.

Machiavelli scrisse "e nelle azioni di tutti li uomini, (...), si guarda al fine", intendendo che sono i risultati che danno valore agli strumenti. Come una caraffa che possiamo riempire con acqua potabile o meno. La scelta è nostra.

Capita a volte che vi sia chi approfitti di questa confusione per nascondersi dietro una notizia o una dinamica comunicativa, sfruttandone il potere che ne deriva.

Manuel Castells descrive perfettamente questo processo nel suo "Comunicazione e potere".

Se la comunicazione è sovranità a noi cogliere questa sfida.

\section{Conclusioni}

Le incertezze di una società che fin dagli anni ' 80 si è presentata -a chi fosse in grado di leggere i dati sensibili- in forte mutamento, devono spingere a porre le questioni europee in maniera più costruttiva. A volte solo impostare un'analisi 
con atteggiamento non pregiudiziale permette di avere una prospettiva chiara.

La condizione storica del continente Europa, uscito da una tragedia fratricida, prima che da una guerra, ha spinto Le Madri e i Padri fondatori, alla fine della Seconda Guerra Mondiale, a pensare ad una struttura protettiva sovranazionale.

Unire e poi tenere insieme gli stati nazionali che cercavano allo stesso tempo di ritrovare un'identità credibile ha fatto sì che si sviluppasse uno scheletro burocratico ipertrofico.

Quelle intenzioni scaturivano da un sacrificio civile e umano molto sentito dalle popolazioni. Si è soprattutto lavorato sulla ricostruzione e si è poco ragionato pubblicamente sull'idea di Federazione Europea come conquista e assicurazione per un futuro di pace.

I pochi attori europei provenienti dalle diverse nazioni, sostenuti da molti silenziosi uomini di pensiero, non hanno mai dubitato che la guerra, con il suo epilogo tragico e la feroce negazione dei diritti umani da cui era scaturita, fosse stata fondante per la nascita delle prime strutture europee. La difficoltà di elaborare nell'intimo gli accadimenti fu per tutti, nessuno escluso (vittime/carnefici/protagonisti/comprimari/spettatori), il limite alla nascita di una Europa politica sin da subito. Si era a quel tempo impegnati nella definizione di una Unione delle Nazioni globale e la miopia di molti governi nazionali, impegnati parallelamente sul fronte interno a risolvere questioni di piccolo e grande cabotaggio, fu un ulteriore ostacolo ad un ragionamento compiuto e analitico sull'idea di Europa Unita. Si ripiegò, in alcuni casi inconsapevolmente, in altri un po' vigliaccamente, su una unione iniziale di tipo commerciale ed economico. Tuttavia negli anni '80 la società dell'informazione venne incontro in qualche modo all'Europa quale mezzo e metodo immateriale per realizzare, come pure fu, un soggetto istituzionale con delle nuove potenzialità e prospettive di unità. Questo ha tuttavia portato con sé degli effetti collaterali, ed oggi quella società dell'informazione si è già trasformata in società della conoscenza, e nel caso dell'Europa si rende necessario un mutamento di protocollo che sia efficace e che riformi le strutture esistenti e le porti al passo con i tempi. Occorrerà ripensare al più presto il concetto di gestione burocratica legato al passato, ormai troppo limitato, alla luce della richiesta da parte dei cittadini stessi che desiderano essere uniti negli intenti, non solo nei passaggi funzionali.

Qui il mondo dei media entra in campo prepotentemente, mostrando ancora troppo poca consapevolezza delle proprie potenzialità comunicative e di formazione.

Infatti ognuno dei comportamenti consapevoli che un cittadino europeo mette in atto deriva da una miscellanea di dati oggettivi, ipotesi, confronti, visioni e costituisce insieme all'eredità di una memoria comune il primo passo verso la costruzione del presente e del futuro europeo. Parole d'ordine nuove e rivoluzionarie possono rimuovere il pregiudizio: puntare al confronto e stimolare il coraggio di appartenere al contesto nel quale si vive.

L'Europa nascerà se nasceranno gli europei, poiché in molti hanno speso e rischiato la propria vita per questo ideale di equilibrio culturale e hanno donato i propri sforzi incondizionatamente per vedere il mondo andare verso una dimensione di intero fatto di parti e di ricchezze uniche nel loro valore.

\section{References}

Castells, M., (2014) Comunicazione e potere, Università Bocconi Ed.

Colombo, G., (2008) Sulle regole, La Feltrinelli.

Colombo, G., (2011) Democrazia, Bollati Boringhieri.

Cooke, M. (2000),"Five Arguments for Deliberative Democracy" in Political Studies; 48: 947-969.

Dahl, R. A. (1989), Democracy and its Critics; New Haven, CT: Yale University Press.

Dryzek, J. S. (2010), Foundations and Frontiers of Deliberative Governance; Oxford: Oxford University Press.

Elster, J. (1998), Deliberative Democracy; Cambridge: Cambridge University Press.

Ferrara, A. (2011), Democrazia e Apertura; Milano: Bruno Mondadori.

Fishkin, J. S. (2011), When the People Speak. Deliberative Democracy \& Public Consultation - Paperback Edition; Oxford: Oxford University Press.

ID. (1997), The Voice of the People: Public Opinion and Democracy - 2. Edition; New Haven, CT: Yale University Press.

ID. (1991), Democracy and Deliberation; New Haven, CT: Yale University Press.

Fishkin, J. S. and Laslett, P. (eds.) (2003), Debating Deliberative Democracy; Malden, MA: Blackwell.

Floridia, A. (2012), La Democrazia Deliberativa: Teorie, Processi e Sistemi; Roma: Carocci Editore.

Gastil, J. and Levin, P. (eds.) (2005), The Deliberative Democracy Handbook: Strategies for Effective Civic Engagement in the 21stCentury; San Francisco, CA: Jossey-Bass.

Goodin, R. E. (2009), Innovating Democracy: Democratic Theory and Practice after the Deliberative Turn; Oxford: Oxford University Press.

Grönlund, K., Bächtiger, A. and Setälä, M. (eds) (2014), Deliberative Mini-Publics. Involving Citizens in the Democratic Process; Colchester: ECPR Press. 
Hobsbawm, J., (2006) II trionfo della borghesia, 1848-1875, Ed. Laterza, IV ed.

Isernia, P. and Fishkin, J. S. (2014), "The EuroPolis Deliberative Poll" inEuropean Union Politics; 15: 1-17.

Kristeva, J., (2014) Stranieri a noi stessi. L'Europa, l'altro, l'identità, nuova ed. accresciuta con una Introduzione, Donzelli Editore.

Machiavelli, N., (1532) II Principe, cap. XVIII.

Minc, A., (1982) L'après-crise est commencé, Paris, Gallimard.

Platone, nel Cratilo parla del "panta rei" di Eraclito, circa 400 a.C.

Rothmann, S., Mosmann, C., (1972) Computers and society: the technology and its social implications, Science Research Associates, Chicago.

Servan-Shreiber, J.J., (1980) La sfida mondiale, Milano.

Sintomer, Y. (2009), Potere al Popolo. Giurie Cittadine, Sorteggio eDemocrazia Partecipativa; Bari: Dedalo.

Smets, K. and Isernia, P. (2014), "The Role of Deliberation in Attitude Change: an Empirical Assessment of Three Theoretical Mechanisms" in European Union Politics; 16: 1-21.

Toffler, A., (1981) The third wave, NY: Banton. www.eur-lex.europa.eu

Gazzetta Ufficiale delle Comunità europee, del 18-12-2000, C 364/10, Libertà di espressione e di informazione, versione IT. www.isaonline.it/mag/UE-Trattati_Protocolli 